\title{
Paramètres de l'endocrinologie sexuelle des béliers Peuls et Touaregs : influence du type génétique et de la saison
}

\author{
M. Issa ${ }^{1}$ H. Marichatou ${ }^{1}$ A. Yenikoye ${ }^{1}$ M. Banoin ${ }^{1}$
}

\begin{abstract}
Mots-clés
Ovin - Bélier Touareg - Bélier Peul Testostérone - LH - Hormone Saison - Niger.
\end{abstract}

\begin{abstract}
Résumé
Les paramètres endocriniens de deux hormones sexuelles [l'hormone lutéinisante ( $\mathrm{LH}$ ) et la testostérone] ont été étudiés chez trois béliers Touaregs et deux béliers Peuls bicolores au cours des mois d'avril et d'octobre 1997. Les prélèvements sanguins ont été effectués de 1 h 00 à 7 h 00 du matin à 15 minutes d'intervalle. Les moyennes ( \pm erreur-type) obtenues pour les différents paramètres étudiés ont été respectivement pour les béliers Peuls et Touaregs : niveaux de base de la testostérone $4,17 \pm 1,82$ et 4,55 $\pm 2,71 \mathrm{ng} / \mathrm{ml}$, et de la $\mathrm{LH} 0,80$ $\pm 0,13$ et $0,96 \pm 0,33 \mathrm{ng} / \mathrm{ml}$; amplitudes des pics de la testostérone 10,16 $\pm 3,11$ et $11,09 \pm 2,82 \mathrm{ng} / \mathrm{ml}$, et de la $\mathrm{LH} \mathrm{1,75 \pm 0,9}$ et $2,65 \pm 1,29 \mathrm{ng} / \mathrm{ml}$; durées des pics de la testostérone $85 \pm 18,7$ et $88,63 \pm 27,47 \mathrm{~min}$, et de la $\mathrm{LH}$ $75 \pm 23,71$ et $61,87 \pm 24,23 \mathrm{~min}$; fréquences des pics de la testostérone 2,38 $\pm 1,3$ et 2,03 $\pm 1,2 \mathrm{pics} / 6 \mathrm{~h}$, et de la LH 1,08 $\pm 0,15$ et 1,85 $\pm 0,97 \mathrm{pics} / 6 \mathrm{~h}$; et durée de l'intervalle entre le pic de $\mathrm{LH}$ et la première élévation significative de la testostérone $26,25 \pm 6,49$ et $31,87 \pm 15,79$ min. Les deux races de béliers n'ont été significativement différentes $(p<0,05)$ qu'au niveau de leur amplitude moyenne de LH. Les béliers Touaregs ont eu une amplitude plus élevée que les béliers Peuls $(2,65 \pm 1,29$ contre 1,75 \pm 0,90 ng/ml). Pour tous les autres paramètres étudiés, la différence entre les deux races n'a pas été significative $(p>0,05)$. La durée du pic de LH a été significativement affectée par la saison $(p<0,05)$, les autres paramètres étudiés ne l'ont pas été. Les évolutions parallèles des niveaux de base de testostérone et de $\mathrm{LH}$ au cours des deux saisons ont mis en évidence l'interaction entre les deux hormones.
\end{abstract}

Chez plusieurs races de béliers de divers milieux, il est établi que la libération dans le sang de l'hormone lutéinisante ( $\mathrm{LH})$ et de la testostérone est caractérisée par des pics : races européennes (Préalpes du Sud et Ile-de-France ; 9, 11), races américaines (Hampshire-Suffolk ; 14 ; et Shropshire et Suffolk ; 6), races australiennes (Mérinos et Corriedale ; 13). Chaque pic de LH est suivi par un pic de testostérone $(12,14)$. Les fréquences des pics de LH et de testostérone augmentent quand les béliers passent de la contre-saison à la saison sexuelle (12).

1. Universite Abdou Moumouni, faculté d'Agronomie, BP 10960, Niamey, Niger. * Auteur pour la correspondance

Tél. : +22793930350; fax : +22720315862

E-mail : mgarbeye@yahoo.fr
En milieu sahélien, notamment au Niger, les profils des hormones sexuelles et leurs variations saisonnières chez les béliers n'ont pas encore été étudiés. Cependant, des études antérieures ont montré que chez la femelle le déterminisme physiologique à la base des variations saisonnières de l'activité sexuelle est étroitement lié à l'évolution du rythme de sécrétion de l'hormone gonadotrope LH (17).

Cette étude a eu pour objectif de déterminer les profils de la LH et de la testostérone ainsi que leur variation saisonnière chez des mâles en milieu sahélien. La LH et la testostérone étant les hormones qui contrôlent le fonctionnement du testicule, de l'épididyme et des glandes annexes, leur dosage pourrait apporter une explication aux taux élevés et faibles des spermatozoïdes morts et anormaux observés respectivement en avril et octobre (7). 


\section{MATERIEL ET METHODES}

\section{Données climatiques}

L'expérience a été conduite dans un parc de l'université de Niamey (latitude $\left.13^{\circ} 30^{\prime} \mathrm{N}\right)$. Les données climatiques fournies par la direction de la météorologie nationale sont caractérisées par : une température moyenne minimale en décembre - janvier $\left(25,7^{\circ} \mathrm{C}\right)$, avec deux pics, l'un en mai $\left(34,4^{\circ} \mathrm{C}\right)$, l'autre en octobre $\left(32^{\circ} \mathrm{C}\right)$; une amplitude thermique plus faible en août $\left(8,4^{\circ} \mathrm{C}\right)$ et maximale en décembre $\left(17,7^{\circ} \mathrm{C}\right)$; un taux d'humidité relative minimal en mars (15,1 p. 100) et maximal en octobre (44,7 p. 100); une durée d'éclairement minimale en décembre (11 h 03$)$ et maximale en mai (12 h 08).

\section{Les animaux}

L'expérience a porté sur trois béliers Touaregs et deux béliers Peuls bicolores. Leur régime alimentaire était composé de paille de riz, de son de riz, de graines de coton et d'oligoéléments sous forme de pierre à lécher. L'eau était distribuée à volonté.

\section{Prélèvements sanguins}

Des prélèvements sanguins en vue du dosage de la testostérone et de la LH ont été effectués en avril et octobre : ces deux mois correspondaient respectivement à des périodes où des taux élevés et faibles de spermatozoïdes morts et anormaux ont été observés (7). En avril et octobre, le sang a été prélevé de 1 h 00 à 7 h 00 du matin à 15 min d'intervalle en évitant de stresser les animaux (16). Le sang a été collecté au niveau de la veine jugulaire et recueilli dans un tube hépariné, puis immédiatement centrifugé au frais à 4000 tours/min pendant $30 \mathrm{~min}$. Le plasma recueilli a été stocké à $-15^{\circ} \mathrm{C}$ jusqu'à la réalisation du dosage radioimmunologique de la testostérone et de la $\mathrm{LH}$.

\section{Dosages hormonaux}

Les échantillons de plasma ont été expédiés au laboratoire de dosages hormonaux de la station de physiologie de la reproduction des mammifères domestiques de l'Inra-Nouzilly en France, où les hormones ont été dosées pour les auteurs en 1998 par la méthode RIA (3).

\section{Détermination des paramètres étudiés}

\section{Niveaux de base moyens des hormones}

La concentration moyenne de l'hormone a été déterminée à partir de toutes les valeurs mesurées pendant la période de prélèvement, c'est-à-dire six heures (15 min d'intervalle) et une bande de confiance (moyenne $\pm 2 \times$ écart-type $/ \sqrt{n} ; n=$ nombre de prélèvements) contenant 95 p. 100 des données (4) a été déterminée chez chaque animal. Les valeurs qui excluaient les pics définis cidessous et qui appartenaient à cet intervalle correspondaient aux niveaux de base moyens.

\section{Amplitudes des pics des hormones}

La sécrétion pulsatile, ou décharge de l'hormone, a été définie par une élévation brutale de la concentration entre l'intervalle de deux prélèvements successifs et a été supérieure à la bande de confiance définie. L'amplitude du pic de l'hormone a été alors déterminée par la concentration maximale mesurée lors de la décharge de l'hormone.

\section{Durée des pics des hormones}

C'est l'intervalle de temps entre la valeur du niveau de base qui a précédé la première élévation significative de l'hormone et la valeur du niveau de base qui a suivi immédiatement la concentration en dehors de la bande de confiance.

\section{Fréquence des pics des hormones}

La fréquence des pics a été définie par le nombre de pics enregistrés par unité de temps ; l'unité de temps a été dans ce cas de six heures de prélèvement.

\section{Sensibilité du testicule à la stimulation de la LH}

C'est l'intervalle de temps entre le point correspondant à la concentration maximale de $\mathrm{LH}$ et le point correspondant à la première élévation significative de testostérone après ce pic de LH.

\section{Analyses statistiques}

Une transformation logarithmique a été effectuée sur l'ensemble des valeurs des différents paramètres hormonaux. Une analyse de variance à deux critères de classification (effet saison et effet race) $(4,15)$ a été utilisée sur ces nouvelles valeurs pour la comparaison des paramètres d'évolution de la testostérone et de la LH.

\section{RESULTATS}

\section{Profil général des hormones}

\section{Testostérone}

Pour tous les deux mois et les deux races de béliers, les pics de testostérone ont été caractérisés par une augmentation progressive de la concentration, suivie d'une diminution également progressive avec souvent l'apparition d'un plateau, ceci ayant eu pour conséquence une augmentation de la durée du pic. L'activité androgénique intense du testicule (pic de testostérone) pouvait apparaître la nuit comme le matin (figures 1 à 5).

Pour les trois béliers Touaregs, sur 11 pics de testostérone définis, neuf ont été précédés par le pic de LH, soit 81,82 p. 100. Chez les béliers Peuls, sur sept pics de testostérone définis, quatre ont été précédés par le pic de LH, soit 57,14 p. 100. Pour les deux races 72,23 p. 100 des pics de testostérone ont été précédés de celui de LH.

\section{LH}

Pour les deux mois et les deux races de béliers, la sécrétion de la LH a été de type pulsatile. Chaque pulse a été caractérisé par une élévation brutale de la concentration entre deux prélèvements successifs. Cette élévation a été suivie d'une diminution de la concentration qui a été relativement rapide. Les durées des pics de LH ont donc été en général plus courtes par rapport à celles des pics de testostérone. L'activité sécrétoire intense de l'hypophyse pour la LH (pic de LH) pouvait aussi apparaître la nuit comme le matin.

\section{Caractéristique des profils hormonaux chez les deux races}

\section{Testostérone}

Aucun des paramètres d'évolution de la testostérone plasmatique étudiés n'a été différent chez les béliers Peuls et les béliers Touaregs $(\mathrm{p}>0,05)$. Les moyennes obtenues respectivement pour les béliers Peuls et Touaregs ont été : niveau de base moyen $(4,17$ $\pm 1,82$ et $4,55 \pm 2,71 \mathrm{ng} / \mathrm{ml})$, amplitude des pics $(10,16 \pm 3,11$ et $11,09 \pm 2,82 \mathrm{ng} / \mathrm{ml})$, durée des pics $(85 \pm 18,7$ et $88,63 \pm 27,47$ $\mathrm{min})$ et fréquence des pics $(2,38 \pm 1,3$ et $2,03 \pm 1,2$ pics $/ 6 \mathrm{~h})$. Cependant, les béliers Touaregs ont eu tendance à avoir des valeurs plus élevées pour ces paramètres que les béliers Peuls bicolores à l'exception de la fréquence des pics (tableau I). 

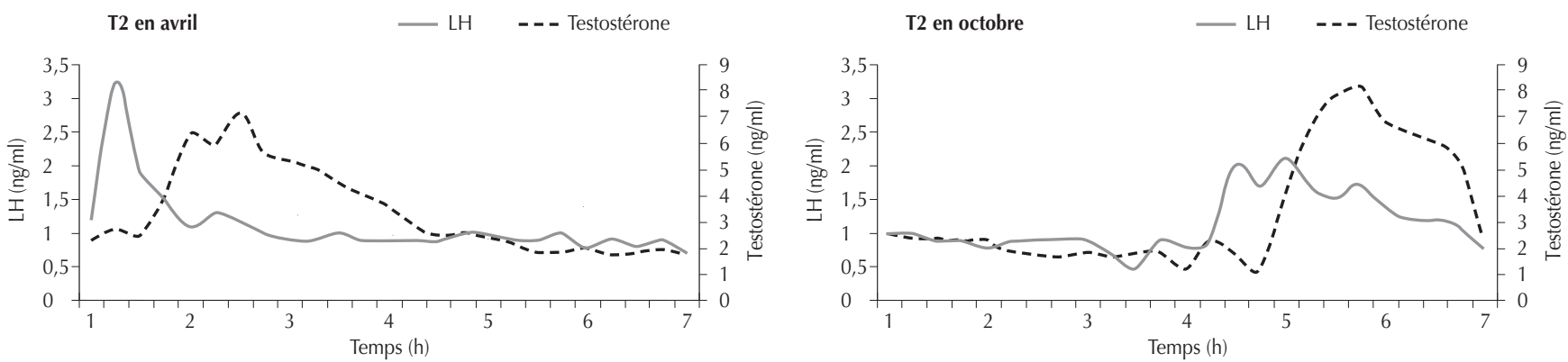

Figure 1 : évolution de la testostérone et de la LH plasmatique chez le bélier Touareg T2.
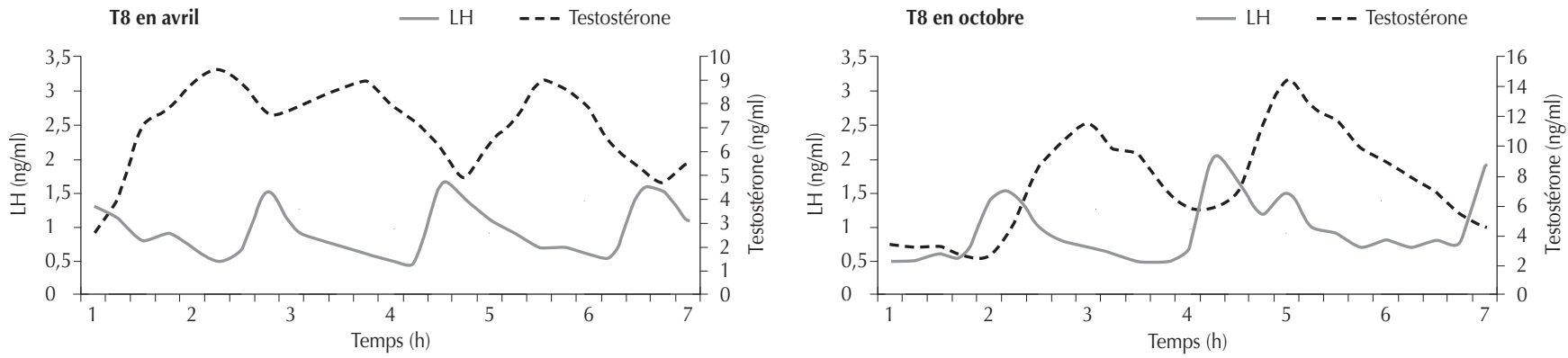

Figure 2 : évolution de la testostérone et de la LH plasmatique chez le bélier Touareg T8.
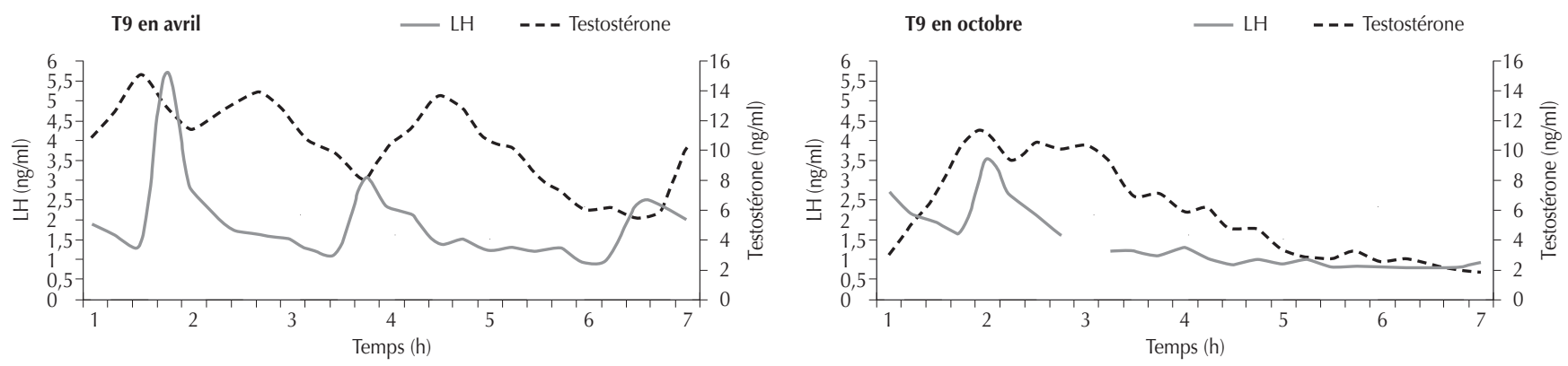

Figure 3 : évolution de la testostérone et de la LH plasmatique chez le bélier Touareg T9.
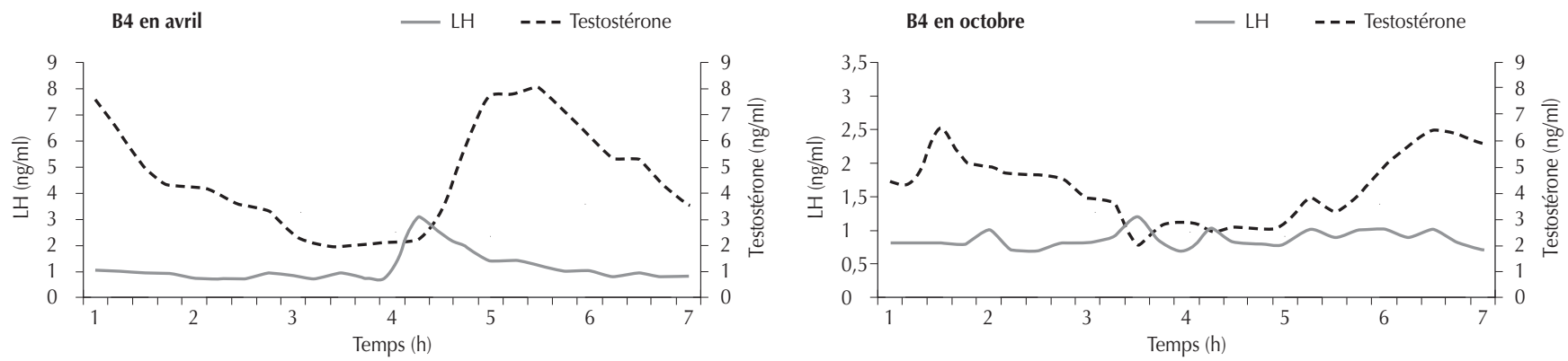

Figure 4 : évolution de la testostérone et de la LH plasmatique chez le bélier Peul B4.
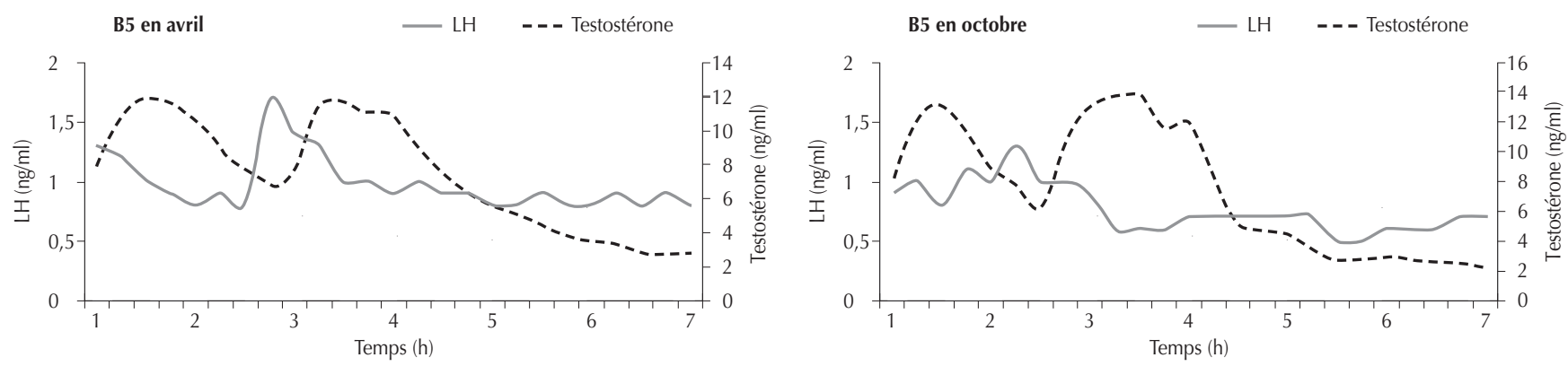

Figure 5 : évolution de la testostérone et de la LH plasmatique chez le bélier Peul B5. 


\section{Tableau I}

Moyennes par race des différents paramètres mesurés (moyenne \pm erreur-type de la moyenne) (valeurs logarithmiques)

\begin{tabular}{|c|c|c|c|}
\hline \multirow{2}{*}{$\begin{array}{l}\text { Hormones } \\
\text { et sensibilité }\end{array}$} & \multirow[t]{2}{*}{ Paramètres mesurés } & \multicolumn{2}{|c|}{ Moyennes par race } \\
\hline & & Béliers Peuls bicolores & Béliers Touaregs \\
\hline \multirow[t]{4}{*}{ Testostérone } & Niveau de base moyen & $4,17 \pm 1,82 \mathrm{ng} / \mathrm{ml}^{\mathrm{a}}$ & $4,55 \pm 2,71 \mathrm{ng} / \mathrm{ml}^{\mathrm{a}}$ \\
\hline & Amplitude moyenne des pics & $10,16 \pm 3,11 \mathrm{ng} / \mathrm{ml}$ a & $11,09 \pm 2,82 \mathrm{ng} / \mathrm{ml}$ a \\
\hline & Durée moyenne des pics & $85 \pm 18,7 \min ^{a}$ & $88,63 \pm 27,47 \min ^{a}$ \\
\hline & Fréquence moyenne des pics & $2,38 \pm 1,3 \mathrm{pics} / 6 \mathrm{~h}^{\mathrm{a}}$ & $2,03 \pm 1,2 \mathrm{pics} / 6 \mathrm{~h}^{\mathrm{a}}$ \\
\hline \multirow[t]{4}{*}{$\mathrm{LH}$} & Niveau de base moyen & $0,8 \pm 0,13 \mathrm{ng} / \mathrm{ml}^{\mathrm{a}}$ & $0,96 \pm 0,33 \mathrm{ng} / \mathrm{ml}^{\mathrm{a}}$ \\
\hline & Amplitude moyenne des pics & $1,75 \pm 0,9 \mathrm{ng} / \mathrm{ml}^{\mathrm{a}}$ & $2,65 \pm 1,29 \mathrm{ng} / \mathrm{ml}^{\mathrm{b}}$ \\
\hline & Durée moyenne des pics & $75 \pm 23,71 \min ^{a}$ & $61,87 \pm 24,23 \min ^{a}$ \\
\hline & Fréquence moyenne des pics & $1,08 \pm 0,15 \mathrm{pic} / 6 \mathrm{~h}^{\mathrm{a}}$ & $1,85 \pm 0,97 \mathrm{pics} / 6 \mathrm{~h}^{\mathrm{a}}$ \\
\hline $\begin{array}{l}\text { Sensibilité du testicule } \\
\text { à l'action de la LH }\end{array}$ & $\begin{array}{l}\text { Duré moyenne de l'intervalle } \\
\text { entre le pic de LH et la } 1^{\text {re }} \text { élévation } \\
\text { significative de testostérone }\end{array}$ & $26,25 \pm 6,49 \min ^{a}$ & $31,87 \pm 15,79 \min ^{a}$ \\
\hline
\end{tabular}

a, b Les valeurs suivies de lettres différentes sont significativement différentes

\section{$L H$}

L'amplitude des pics de LH a été significativement plus élevée $(\mathrm{p}<0,05)$ chez les béliers Touaregs que chez les béliers Peuls $(1,75$ $\pm 0,9$ contre $2,65 \pm 1,29 \mathrm{ng} / \mathrm{ml}$ ) (tableau I). Le niveau de base de la $\mathrm{LH}$, la durée des pics et leur fréquence n'ont pas été significativement différents entre les deux races $(p>0,05)$. Les moyennes obtenues pour les béliers Peuls et Touaregs ont été respectivement : niveau de base $0,8 \pm 0,13$ et $0,96 \pm 0,33 \mathrm{ng} / \mathrm{ml}$, durée des pics $75 \pm 23,71$ et $61,87 \pm 24,23 \mathrm{~min}$, fréquence des pics $1,08 \pm 0,15$ et $1,85 \pm 0,97$ pics $/ 6 \mathrm{~h}$ (tableau I).

\section{Sensibilité du testicule à la stimulation de la $\mathrm{LH}$}

La réponse du testicule à la stimulation de l'hormone hypophysaire n'a pas été significativement différente entre les deux races de béliers ( $p>0,05)$. Les moyennes obtenues pour la durée moyenne de l'intervalle entre le pic de $\mathrm{LH}$ et la première élévation significative de la testostérone ont été : 26,25 $\pm 6,49$ min pour les béliers Peuls et $31,87 \pm 15,79$ pour les béliers Touaregs.

\section{Sensibilité des paramètres hormonaux aux variations saisonnières}

\section{Testostérone}

Pour chacune des deux races de béliers, le niveau de base moyen de testostérone n'a pas été différent significativement entre le mois d'avril et celui d'octobre ( $p>0,05)$ (tableau II). L'interaction race - mois n'a pas été non plus significative ( $p>0,05)$. L'amplitude des pics, leur durée moyenne et leur fréquence moyenne n'ont pas varié significativement $(\mathrm{p}>0,05)$ entre les mois d'avril et d'octobre (tableau II). Cependant l'interaction race - mois a été significative $(\mathrm{p}<0,05)$ pour l'amplitude des pics.

\section{LH}

Pour chacune des deux races de béliers, la durée des pics de LH a été significativement plus élevée en octobre qu'en avril $(\mathrm{p}<0,05)$ (tableau II). Le niveau de base de la LH, l'amplitude des pics, leur durée et leur fréquence n'ont pas été affectés par le mois de prélèvement $(\mathrm{p}>0,05)$.

\section{Délai de réponse du testicule à l'action de la $\mathrm{LH}$}

Le délai de réponse du testicule à l'action de la LH n'a pas été différent de façon significative $(\mathrm{p}>0,05)$ entre les mois d'avril et d'octobre. Cependant une tendance au raccourcissement du délai de réponse a été observée en octobre chez les deux races (tableau II). L'interaction race - mois a été significative.

\section{DISCUSSION}

Le coût du dosage des hormones avait justifié la durée relativement courte $(6 \mathrm{~h})$ des prélèvements sanguins et le faible nombre d'animaux utilisés au cours de cette étude. Des prélèvements de 15 minutes d'intervalle pendant six heures ont été également préférés à des prélèvements horaires qui auraient pu masquer des élévations subites et brutales de la LH dont le processus de sécrétion est de type pulsatile $(12,14,16)$. Ainsi, avec six heures de prélèvements le protocole ne peut mettre en évidence d'éventuelles variations des pulses de ces hormones au cours du nycthémère, comme l'ont montré Ortavant et coll. (9) sur les béliers, et Thibier (16) sur le taurillon.

Chez les deux races de béliers, 72,23 p. 100 des pics de testostérone ont eu lieu après le pic de LH ; ceci était comparable aux résultats observés chez d'autres races de béliers européens (Préalpes du Sud et Ile-de-France : 97,2 p. 100) $(9,12)$. Ainsi les profils des deux hormones ont été semblables à d'autres : à ceux de races européenne [Suffolk (8)], américaine [Hampshire-Suffolk (14), et Shropshire et Suffolk (6)], australienne [Merinos et Corriedale (5, 13)], et à celui du veau (11). Les évolutions parallèles des niveaux de base de testostérone et de LH, qui ont été indépendantes des saisons (11), ont mis en évidence l'interaction entre les deux hormones.

Les moyennes des différents paramètres de sécrétion de la testostérone (niveau de base, amplitude, durée et fréquence des pics) n'ont pas été significativement différentes entre ces deux races de béliers ; cependant, en milieu tempéré Boland et coll. (2) ont montré que le niveau de testostérone variait avec les races. 


\section{Tableau II}

Variations des paramètres endocriniens en valeurs logarithmiques en avril et octobre chez les deux races de béliers (moyenne \pm erreur-type de la moyenne)

\begin{tabular}{|c|c|c|c|c|c|}
\hline \multirow[t]{2}{*}{ Hormones } & \multirow[t]{2}{*}{ Paramètres mesurés } & \multicolumn{2}{|c|}{ Avril } & \multicolumn{2}{|c|}{ Octobre } \\
\hline & & Peuls bicolores & Touaregs & Peuls bicolores & Touaregs \\
\hline Testostérone & $\begin{array}{l}\text { Niveau de base moyen } \\
\text { Durée moyenne des pics } \\
\text { Amplitude moyenne des pics } \\
\text { Fréquence moyenne des pics }\end{array}$ & $\begin{array}{c}4,26 \pm 1,79 * \mathrm{ng} / \mathrm{ml}^{\mathrm{a}} \\
95 \pm 7,1 * \mathrm{~min}^{\mathrm{a}} \\
10,47 \pm 1,75 * \mathrm{ng} / \mathrm{ml}^{\mathrm{a}} \\
2,3 \pm 1,1 * \mathrm{pics} / 6 \mathrm{~h}^{\mathrm{a}}\end{array}$ & $\begin{array}{l}5,40 \pm 3,04 * \mathrm{ng} / \mathrm{ml}^{\mathrm{a}} \\
75 \pm 19,6 * \mathrm{~min}^{\mathrm{a}} \\
10,99 \pm 2,8 * \mathrm{ng} / \mathrm{ml}^{\mathrm{b}} \\
2,6 \pm 1,1 * \mathrm{pics} / 6 \mathrm{~h}^{\mathrm{a}}\end{array}$ & $\begin{array}{c}4,10 \pm 1,83 * \mathrm{ng} / \mathrm{ml}^{\mathrm{a}} \\
75 \pm 21,2 * \mathrm{~min}^{\mathrm{a}} \\
9,96 \pm 3,48 * \mathrm{ng} / \mathrm{ml}^{\mathrm{a}} \\
2,45 \pm 1,1 * \mathrm{pics} / 6 \mathrm{~h}^{\mathrm{a}}\end{array}$ & $\begin{array}{c}3,79 \pm 2,11 * \mathrm{ng} / \mathrm{ml}^{\mathrm{a}} \\
108,75 \pm 26,8 * \mathrm{~min}^{\mathrm{a}} \\
11,28 \pm 2,27 * \mathrm{ng} / \mathrm{ml}^{\mathrm{b}} \\
1,5 \pm 0,7 * \mathrm{pics} / 6 \mathrm{~h}^{\mathrm{a}}\end{array}$ \\
\hline $\mathrm{LH}$ & $\begin{array}{l}\text { Niveau de base moyen } \\
\text { Durée moyenne des pics } \\
\text { Amplitude moyenne des pics } \\
\text { Fréquence moyenne des pics }\end{array}$ & $\begin{array}{c}0,87 \pm 0,1 * \mathrm{ng} / \mathrm{ml}^{\mathrm{a}} \\
75 \pm 15 * \mathrm{~min}^{\mathrm{a}} \\
2,35 \pm 0,65 * \mathrm{ng} / \mathrm{ml}^{\mathrm{a}} \\
1,15 \pm 0,15 * \mathrm{pic} / 6 \mathrm{~h}^{\mathrm{a}}\end{array}$ & $\begin{array}{c}1,06 \pm 0,4 * \mathrm{ng} / \mathrm{ml}^{\mathrm{a}} \\
51 \pm 7,34 * \mathrm{~min}^{\mathrm{a}} \\
2,9 \pm 1,4 * \mathrm{ng} / \mathrm{ml}^{\mathrm{a}} \\
2,23 \pm 0,9 * \mathrm{pics} / 6 \mathrm{~h}^{\mathrm{a}}\end{array}$ & $\begin{array}{c}0,73 \pm 0,11 * \mathrm{ng} / \mathrm{ml}^{\mathrm{a}} \\
75 \pm 30 * * \mathrm{~min}^{\mathrm{a}} \\
1,15 \pm 0,15 * \mathrm{ng} / \mathrm{ml}^{\mathrm{a}} \\
1 \pm 00 * \mathrm{pic} / 6 \mathrm{~h}^{\mathrm{a}}\end{array}$ & $\begin{array}{l}0,86 \pm 0,21 * \mathrm{ng} / \mathrm{ml}^{\text {a }} \\
80 \pm 30,82 * * \min ^{\text {a }} \\
2,24 \pm 0,74 * \mathrm{ng} / \mathrm{ml}^{\text {a }} \\
1,5 \pm 0,7 * \mathrm{pics} / 6 \mathrm{~h}^{\text {a }}\end{array}$ \\
\hline $\begin{array}{l}\text { Sensibilité } \\
\text { du testicule } \\
\text { à l'action } \\
\text { de la LH }\end{array}$ & $\begin{array}{l}\text { Durée moyenne de } \\
\text { l'intervalle entre le pic } \\
\text { de LH et la } 1^{\text {re }} \text { élévation } \\
\text { significative de testostérone }\end{array}$ & $30 \pm 00 * \min ^{a}$ & $39 \pm 12 * \min ^{b}$ & $22,5 \pm 7,5^{*} \min ^{a}$ & $20 \pm 14,14 * \min ^{b}$ \\
\hline
\end{tabular}

a, b Au niveau du mois, les valeurs suivies de lettres différentes sont significativement différentes

* et ** Entre avril et octobre et pour une même race les valeurs suivies de * sont significativement différentes de celles suivies de **

Chez les deux races, seule la durée moyenne du pic de LH a subi une variation saisonnière significative. Elle a été plus élevée en octobre qu'en avril avec un niveau de base qui n'a pas été différent entre les deux mois. Plusieurs études montrent qu'aux latitudes élevées, le facteur environnemental qui contrôle le niveau de sécrétion de la LH et par suite celui de la testostérone est la photopériode. Une diminution de la photopériode coïncide avec une élévation du niveau de sécrétion de la LH et de la testostérone $(1,6,10)$. Dans les conditions expérimentales de la présente étude, les variations de la photopériode ne dépassant pas $1,30 \mathrm{~h}$ environ, les températures élevées d'octobre ont probablement contribué à un prolongement de la durée du pic de LH tout en maintenant faibles les niveaux hormonaux de ces deux races par rapport à ceux observés aux latitudes élevées $(6,12,14)$. Des travaux antérieurs ont déjà mis en évidence chez la brebis Peule bicolore une chute du niveau de LH en septembre/octobre en réponse à une augmentation de la température à cette période de l'année sous climat sahélien (18).

\section{CONCLUSION}

Il ressort de cette étude que les caractéristiques d'évolution de la testostérone ont été semblables chez les deux races. L'évolution de la LH a été également semblable chez les deux races à l'exception de l'amplitude des pics qui a été significativement plus élevée chez les béliers Touaregs. La durée des pics a montré aussi une variation saisonnière significative. Les évolutions parallèles des niveaux de base de testostérone et de LH au cours des deux saisons ont mis en évidence l'interaction entre les deux hormones.

\section{Remerciements}

Les auteurs tiennent à remercier M. D. André, responsable du laboratoire de dosages hormonaux, et ces collaborateurs, à la station de physiologie de la reproduction des mammifères domestiques (Inra-Nouzilly, France), qui ont dosé pour nous gratuitement ces hormones en 1998.

\section{BIBLIOGRAPHIE}

1. ALBERIO R., 1976. Rôle de la photopériode dans le développement de la fonction de reproduction chez I'agneau Ile-de-France, de la naissance à 21 mois. Thèse Doct., université de Paris VI, France.

2. BOLAND M.P., AL-KAMALI A.A., CROSBY T.F., HAYNES N.B., HOWLES C.M., KELLEHER D.L., GORDON I., 1985. The influence of breed, season and photoperiod on semen characteristics, testicular size, libido and plasma hormone concentrations in rams. Anim. Reprod. Sci., 9: $241-252$

3. CANEPA S., GAUTHIER C., ANDRE D., 1996. Dosage RIA de la LH ovine dans le plasma et les milieux de culture. Tours, France, Inra, 51 p.

4. DAGNELIE P., 1975. Théorie et méthodes statistiques, vol. 2 Gembloux, Belgique, Les presses agronomiques, 463 p.

5. FERNANDEZ-ABELLA A., BECU-VILLABOS D., LACAU-MENGIDOO I.M., VILLEGAS N., BENTANCUR O., 1999. Sperm production, testicular size, serum gonadotropins and testosterone levels in Merino and Corriedale breeds. Reprod. Nutr. Develop., 39: 617-624.

6. FOSTER D.L., MICKELSON I.H., RYAN K.D., COON G.A., DRONGOWSKI R.A., HOLT J.A., 1978. Ontogeny of pulsatile luteinizing hormone and testosterone secretion in male lambs. Endocrinology, 102: $1137-1146$

7. ISSA M., YENIKOYE A., MARICHATOU H., BANOIN M., 2001. Spermogramme de béliers Peuls bicolores et Touaregs : influence du type génétique et de la saison. Revue Elev. Méd. vét. Pays trop., 54 : 269-275.

8. KATONGOLE C.B., NAFTOLI N., SHORT R.V., 1974. Seasonal variations in blood luteinizing hormone and testosterone levels in rams. J. endocrinol., 60: 101-106.

9. ORTAVANT R., DAVEAU A., GARNIER D.H., PELLETIER J., DE REVIERS M.M., TERQUI M., 1982. Diurnal variation in release of LH and testosterone in the ram. J. Reprod. Fertil., 64: 347-353.

10. PELLETIER J., ALMEIDA G., 1987. Short light cycles induce persistent reproductive activity in Ile-de-France rams. J. Reprod. Fertil. (suppl.), 34 215-226.

11. PELLETIER J., CARREZ-CAMOUS S., THIERY J.C., 1981. Basis neuroendocrine events before puberty in cattle, sheep and pigs. $/$ Reprod. Fertil. (suppl.), 30: 91-102. 
12. PELLETIER J., GARNIER D.H., DE REVIERS M.M., TERQUI M. ORTAVANT R., 1982. Seasonal variations in LH and testosterone release in rams of two breeds. J. Reprod. Fertil., 64: 341-346.

13. PEREZ R.C., FORSBERG M., MARTINEZ H.R., 1998. Seasonal variation in live weight, testes size, testosterone, $\mathrm{LH}$ secretion, melatonin and thyroxine in Merino and Corriedale rams in a subtropical climate. Acta vet. Scand., 39: 35-47.

14. SCHANBACHER B.D., FORD J.J., 1976. Seasonal profiles of plasma luteinizing hormone, testosterone and oestradiol in the ram. Endocrinology, 99: 752-757.

15. SCHWARTZ D., 1983. Théorie et méthodes statistiques à I'usage des médecins et des biologistes, $3^{\mathrm{e}}$ édn. Paris, France, Flammarion médecinesciences, $125 \mathrm{p}$.
16. THIBIER M., 1975. Variations circadiennes de la LH plasmatique chez le jeune taurillon. Ann. Endocrinol., 36 : 205-210.

17. YENIKOYE A., 1991. Déterminisme physiologique des variations saisonnières de l'activité ovarienne chez la brebis en milieu sahélien. In: Proc. International symposium on nuclear and techniques in animal production and health, jointly organized by IAEA and FAO, Vienna, Austria, 15-19 Apr. 1991, p. 391-403.

18. YENIKOYE A., RAVAULT J.P., 1982. Rythme circannuel de sécrétion de la prolactine chez la brebis Peulh. C.R. Acad. Sci., 293, série III : 523525 .

Reçu le 24.03.2006, accepté le 09.05.2007

\section{Summary}

Issa M., Marichatou H., Yenikoye A., Banoin M. Sexual/Endocrine Parameters in Peul and Tuareg Rams: Genetic Type and Seasonal Influence

Endocrinal parameters of two sexual hormones [luteinizing hormone (LH) and testosterone] were studied in three Tuareg rams and two bicolor Peul rams during April and October 1997. Blood samples were collected at 15 min intervals from 1 h00 to 7 h00 AM. Means ( \pm standard error) obtained for the various parameters studied in Peul and Tuareg rams were as follows, respectively: basal levels of testosterone $4.17 \pm 1.82$ and $4.55 \pm 2.71 \mathrm{ng} / \mathrm{ml}$, and $\mathrm{LH} 0.80 \pm 0.13$ and $0.96 \pm 0.33$ $\mathrm{ng} / \mathrm{ml}$; peaks of testosterone $10.16 \pm 3.11$ and $11.09 \pm 2.82$ $\mathrm{ng} / \mathrm{ml}$, and LH $1.75 \pm 0.90$ and $2.65 \pm 1.29 \mathrm{ng} / \mathrm{ml}$; duration of peaks of testosterone $85 \pm 18.7$ and $88.63 \pm 27.47 \mathrm{~min}$, and LH $75 \pm 23.71$ and $61.87 \pm 24.23 \mathrm{~min}$; frequency of peaks of testosterone $2.38 \pm 1.3$ and $2.03 \pm 1.2$ peaks/6h, and LH 1.08 \pm 0.15 and $1.85 \pm 0.97$ peaks $/ 6 \mathrm{~h}$. Time intervals between the peak of $\mathrm{LH}$ and the first significant rise of testosterone were $26.25 \pm 6.49$ and $31.87 \pm 15.79$ min, respectively. The only significant $(p<0.05)$ differences between the two breeds were their average amplitude of $\mathrm{LH}$. Tuareg rams had an amplitude higher than that of Peuls $(2.65 \pm 1.29 \mathrm{ng} / \mathrm{ml}$ vs $1.75 \pm 0.90$ $\mathrm{ng} / \mathrm{ml}$ ). For all the other parameters studied, the differences between the two breeds were not significant $(p>0.05)$. The duration of the peak of $\mathrm{LH}$ was significantly affected by the season $(p<0.05)$, whereas the other parameters were not. Parallel evolutions of the basal levels of testosterone and LH during both seasons highlighted the interaction between the two hormones.

Keywords: Sheep - Tuareg ram - Peul ram - Testosterone - LH Hormone - Season - Niger.

\section{Resumen}

Issa M., Marichatou H., Yenikoye A., Banoin M. Parámetros de la endocrinología sexual de los corderos Peuls y Tuareg: influencia del tipo genético y de la estación

Se estudiaron los parámetros endocrinos de dos hormonas sexuales [hormona luteinizante $(\mathrm{LH})$ y la testosterona] en tres corderos Tuareg y dos corderos Peuls bicolores, durante el curso de los meses de abril y octubre 1997. Las muestras sanguíneas se efectuaron de la 1:00 a las 7:00 de la mañana con 15 minutos de intervalo. Los promedios obtenidos para los diferentes parámetros estudiados fueron, respectivamente para los corderos Peuls y Tuareg: niveles de base de la testosterona $4,17 \pm 1,82$ y $4,55 \pm 2,71 \mathrm{ng} / \mathrm{ml}$, y de la $\mathrm{LH} 0,80$ $\pm 0,13$ y $0,96 \pm 0,33 \mathrm{ng} / \mathrm{ml}$; picos de amplitud de la testosterona $10,16 \pm 3,11$ y $11,09 \pm 2,82 \mathrm{ng} / \mathrm{ml}$, y de la $\mathrm{LH} 1,75$ $\pm 0,9$ y $2,65 \pm 1,29 \mathrm{ng} / \mathrm{ml}$; duración de los picos de la testosterona $85 \pm 18,7$ y $88,63 \pm 27,47 \mathrm{~min}$, y de la LH $75 \pm 23,71$ et $61,87 \pm 24,23 \mathrm{~min}$; frecuencias de los picos de la testosterona $2,38 \pm 1,3$ y $2,03 \pm 1,2$ picos/ $6 \mathrm{~h}$, y de la LH 1,08 $\pm 0,15$ y $1,85 \pm 0,97$ picos/ 6 h; y duración del intervalo entre el pico de $\mathrm{LH}$ y el primer aumento significativo de la testosterona $26,25 \pm 6,49$ y $31,87 \pm 15,79 \mathrm{~min}$. Las dos razas de corderos no fueron significativamente diferentes $(p<0,05)$, sino a nivel de la amplitud media de LH. Los corderos Tuareg mostraron una amplitud más elevada que los corderos Peuls $(2,65 \pm 1,29$ contra $1,75 \pm 0,90 \mathrm{ng} / \mathrm{ml}$ ). Para todos los otros parámetros estudiados, la diferencia entre las dos razas no fue significativa ( $p>0,05)$. La duración del pico de LH fue significativamente afectada por la estación ( $p<0,05)$, los otros parámetros estudiados no lo fueron. Las evoluciones paralelas de los niveles de base de la testosterona y de la LH durante el curso de las dos estaciones pusieron en evidencia la interacción entre las dos hormonas.

Palabras clave: Ovino - Morueco Tuareg - Morueco Peul Testosterona - LH - Hormona - Estación del año - Níger. 